\title{
Coding, causality, and statistical craft: The emergence and evolutionary drivers of moralistic supernatural punishment remain unresolved
}

\author{
Benjamin Grant Purzycki*a, Theiss Bendixen ${ }^{\mathrm{a}}$, Aaron D. Lightner ${ }^{\mathrm{a}}$ \\ ${ }^{a}$ Department of the Study of Religion, Aarhus University, DK
}

\section{Introduction}

The target article represents another attempt to assess the relationship between social complexity and moralistic supernatural punishment. This question is important and the authors are to be commended for their continued commitment to a more open science. However, the target article includes some sizeable issues related to the contention that the authors' initial attempt "was critiqued [in Beheim et al. 2021] on the grounds that little could be known about prehistoric beliefs in Big Gods". To be sure, Beheim et al.'s critique was multi-faceted, but its premises did not include the point that the past is effectively unknowable. Rather, it demonstrated that the initial attempt's results were inextricably linked to the decision to replace missing data with 0 (i.e., absent), a decision that led to the paper's retraction (Whitehouse et al., 2021). McElreath (2020) uses the first attempt to illustrate how the research question is not reliably testable given the way the data were generated and how this process biases any analysis of the data. The current iteration in the target article compounds the problem; since the retraction, the Seshat database has replaced the bulk of its missing values of moralistic supernatural punishment with "inferred absences". These "inferred absences" are still inextricably linked to the kind of data included in the database.

In our evaluation of the project outlined in the target article, we must conclude that at each step-from data production and theory to modeling and reporting-the current target article's workflow actually makes it impossible to test the hypothesis its authors claim they are testing. At best, the target article describes the pertinent Seshat data in its present state, but it does not and cannot make sound inferences about the target causal historical hypothesis. While we have identified many other

\footnotetext{
${ }^{*}$ Corresponding author email: bgpurzycki@cas.au.dk. $\mathrm{R}$ code for this commentary can be found at: https://github.com/alightner/seshatCommentary2022.
} 
problems with source material, operational assumptions, analysis, and conception, we restrict our discussion to three important classes of issues: problems of data, analysis, and causal inference.

\section{Measurement, validity, and the ethnographic record}

The data and its codes neither reflect the ethnographic world nor the precedent constructs designed to address the question of when "moralistic gods" ("high" or otherwise) evolved. Setting aside the classical anthropological literature that emphasizes the presence of moralistic gods in traditional societies (e.g., Evans-Pritchard, 1965; Lang, 1909; Malinowski, 1936), contemporary group-level studies (Boehm, 2008; Swanson, 1960; Watts et al., 2015) show non-trivial and even high proportions of small-scale societies having moralistic supernatural punishment. Individual-level cross-cultural projects motivated by the extant target hypotheses (Bendixen et al., nd; Purzycki, 2011, 2013; Purzycki et al., 2022; Singh et al., 2021) also point to the presence of moralistic supernatural punishment in traditional religions. In some reports, such beliefs are detectable even after holding constant the correlation with moralistic traditions. In sum, when looking at the ethnographic record or directly engaging in ethnographic work, one finds that there is considerable and striking evidence that small-scale societies have moralistic supernatural punishment.

The retracted article replaced missing values with "absence" at the analysis stage of its workflow. Now, the missing values have been largely replaced with "inferred absences" in the database itself, thus tainting any subsequent empirical project at its source. Populating Seshat's missing data with "inferred absence" is neither based on the ethnographic, historical, and archaeological records, nor on some principled prior-defined probability distribution. Importantly, to the best of our accounting, there does not appear to be a single written record in the Seshat database that documents moralistic gods among small-scale societies prior to ethnography. In fact, we're offered no vision of what this-or any other kind of evidence-would look like among such populations. Indeed, the moralistic traditions are overwhelmingly Christian, Buddhist, Hindu, and Muslim where copious amounts of documentation (and common assumptions) are available for use. Here, then, we have a historical process mechanism that drives the availability of actual data. Here too, then, all results are therefore descriptions of the available data in the database, and not inferences about the past. By way of general principle, then: if a historical database replaces missing values with data and this data is correlated with time and/or a mechanism of recording information, any analysis will inevitably find a relationship with time and/or that mechanism of recording information. This was the thrust of 
the aforementioned critiques (Beheim et al., 2021; McElreath, 2020, see below).

Yet, even the target article's data handling procedures inflate the magnitude of the result. Turchin et al. code the presence/absence of seven moralistic supernatural punishment (MSP) features for each polity. By itself, disaggregating MSP is a fine idea; MG features (and their representation across sources) often vary, and we can enrich our understanding of MSP with both fine- and coarse-grained analyses available. It is less obvious, however, why they weight each added MSP feature on a multiplicative scale, where each additional feature has double the impact of the previous one (as outlined in and around Table 1 of the target article).

To illustrate what their assumption implies, suppose that at a given time step, Culture A has one known MSP feature and Culture B has six. If we see evidence for one additional MSP feature at the next time step in Cultures A and B, then Turchin et al.'s multiplicative MSP scale would weight Culture B's additional MSP feature as 32 times "more present" than Culture A's. We know of no rationale for this, and the target article does not provide one. Setting aside how we might interpret missing MSP features in a given polity, this scaling decision forces an MSP variable with many intermediate values into a bimodal one, where "most polities are characterized by low values (MSP $<0.2)$, or by relatively high values $(>0.8)$, with a few values in between" (sect. 3.2). Indeed, we can see that their scale widens the divide between polities with more than six features versus polities with four or fewer features.

The premise of Turchin et al.'s article - a "rise of moralizing religions" in recent human history - is visualized in Figure 1 of the target article, which includes two "hotspots": a low MSP hotspot (c. 500 BCE) followed shortly after with a high MSP hotspot (c. $1000 \mathrm{CE}$ ). This dramatic upswing in the prevalence of MSP, however, is highly dependent on the multiplicative MSP scale, which compresses cases with fewer MSP features into the same hotspot as cases with no known MSP features. As we show in the upper panel of Figure 1 below, switching from the arbitrary multiplicative MSP scale to a simpler additive one causes the lower hotspot to disaggregate, suggesting a less distinct pattern over time.

We should reemphasize that more vs. fewer MSP features does not imply more vs. less MSP for each polity. Rather, they imply more vs. less available evidence for MSP - an availability that only degrades as we look further back in time. A simpler alternative to splicing MSP features and debating about how we should merge them together would be to examine the presence vs. absence of evidence for MSP in each polity. When we plot this across time, it becomes much clearer that our ability to see MSP in the past is almost exclusively limited to how far back we 

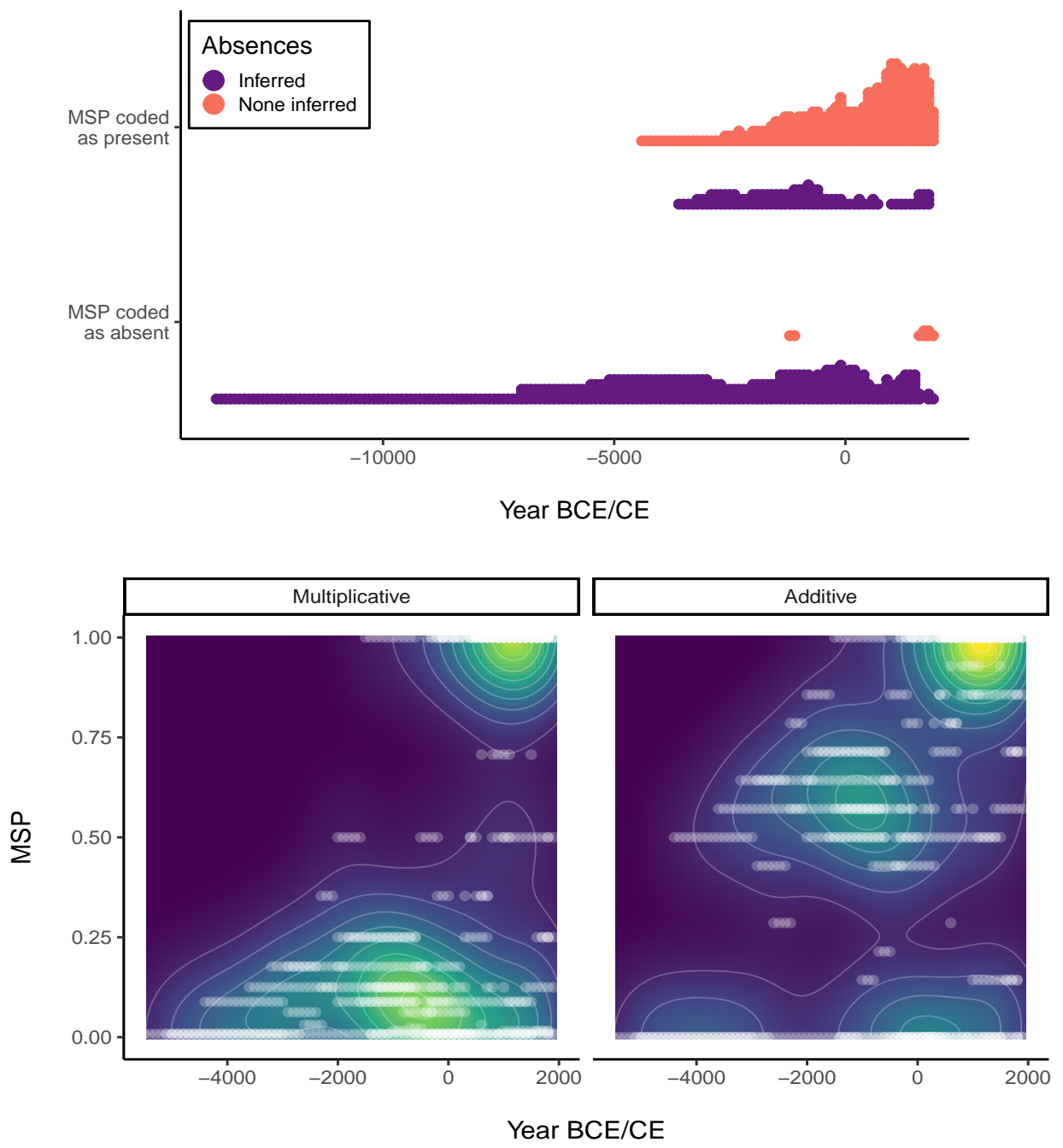

Figure 1: Upper: Seshat polities with evidence for MSP present (upper) vs. absent (lower) distributed across time. Presence vs. absence of MSP evidence is broken down by whether or not the coding of MSP evidence includes any inferred absences. Lower: Change in MSP in sample regions over time, based on Seshat dataset linked to the target article. The dots are a scatter plot of MSP against time (negative values are $\mathrm{BCE}$, positive values are $\mathrm{CE}$ ). The left panel uses the multiplicative MSP variable to replicate Figure 1 in the target article, where light areas and their contours delineate high density hotspots. The right panel replicates the left panel, but uses an additive MSP variable instead of a multiplicative one. 
can see into history. ${ }^{2}$ The presence of evidence for MSP reaches quite far into the past, and almost all of the instances where evidence for MSP are coded as "absent" result from inferred absences (463 out of 471 observations where MSP is said to be absent). This strongly suggests that the absences of MSP almost entirely rely on the assumption that an absence of an MSP feature at Time X implies it is also absent at all previous times (see lower panel of Figure 1). This inevitably provides an answer to the very question the authors wish to test. How, then, do the tests fare?

\section{3. "Significance" and collinearity}

While we identified a range of analytical issues, we focus here on some of the pitfalls of statistical "significance" and collinearity in the context of causal inquiry. ${ }^{3}$ Inspecting the pairs plot (Fig. S2 in the target article supplements), the distributional overlap between key variables - MilTech, Cavalry, and SPC - is striking. All three are also strongly associated with MSP. This is a potential source of collinearity, whereby the (conditional) association between predictor variables can mask otherwise "true" associations. For instance, when predicting MSP (Table 2-3 in the target article), SPC is excluded on the grounds that it is not a "significant" predictor term in alternative model specifications. This result is consistent with masking effects between MilTech, Cavalry, and SPC: since the model already includes MilTech and Cavalry, we do not learn much by adding SPC, given that much of the relevant information in SPC is also in MilTech and Cavalry. This suspicion is testable: does SPC gain in associational strength if MilTech and Cavalry are excluded when predicting MSP? The answer is "yes" (see R script for a simple re-analysis). Further, in the model predicting SPC, a large overlap between MilTech and Cavalry (Table 4 in the target article) - combined with the autoregression terms - might leave very little variation to be explained by MSP. This, too, is testable: does MSP gain in associational strength if MilTech, Cavalry, and autoregression terms are excluded

\footnotetext{
${ }^{2}$ This is the "streetlight effect," an observational bias that virtually always limits our ability to make inferences based on historical and archaeological data.

${ }^{3}$ Others worth considering further include sub-optimal modeling choices. Index scores (e.g., the MSP variable), which are zero-one inflated and bounded, rarely satisfy common assumptions in linear regression but call for alternative likelihood specifications. Modeling index scores as metric might severely under- or over-estimate a target effect (e.g., Ospina and Ferrari, 2012; Liu and Kong, 2015). Moreover, AIC is being supplanted by contemporary alternatives, such as WAIC (Gelman et al., 2014; Watanabe, 2010) and approximate leave-one-out cross-validation (Vehtari et al., 2017) that simultaneously relax restricting assumptions and that are more widely applicable (cf., McElreath, 2020).
} 
when predicting $S P C$ ? The answer is, again, "yes". Of course, this latter model goes against the authors' hypothesis that a possible relationship between SPC and MSP is spurious and induced by a common cause, or a "confounder", namely military competition (MilTech and Cavalry).

More importantly, whether a variable should be considered a confounder depends not on the data, but on the causal graph or, more generally, on explicit prior assumptions of the data-generating process (e.g., Hernán et al., 2002). Causal inference and prediction are two distinct statistical tasks; however, the authors confuse the two throughout their analysis. While the explicit goal of the study is to elicit "drivers" and "causes" of social complexity (SPC) and moralizing supernatural punishment (MSP), the authors rely on model comparison metrics (Akaike Information Criterion/AIC, and $R^{2}$ ) to determine the better causal story. These statistics might gauge in- or out-of-sample predictive accuracy, but they are wholly unfit for causal inference (cf., McElreath, 2020). In fact, AIC and $R^{2}$ favor confounded models. Therefore, systematically excluding variables on the basis of models' AIC scores (or $p$-values) brings us no closer to detecting causal relationships - particularly in the presence of autoregression terms, which will naturally increase predictive accuracy (for further details, see $\mathrm{R}$ script). Inferring causality requires more than just data. It requires explicit causal assumptions of the data-generating process. We turn to this point now.

\section{Causal inference without a causal model}

It is a basic and fundamental premise in causal inference that statistical choices are guided by explicit causal assumptions of the data-generating process prior to analysis - for instance in the form of a directed acyclic graph (DAG) informed by previous studies and/or domain expertise (see Pearl et al., 2016). In contrast, the authors build their causal graph (Fig. 3 in the target article) post-analysis on the basis of regression coefficients, $p$-values, and model comparison metrics. As discussed, this is problematic since the direction of causality cannot be determined by data (or regression coefficients) alone. With reference to their correlational pairs plots (Fig. $\mathrm{S} 2$ ), the authors acknowledge that "correlation analysis cannot reveal causal interconnection" (p. 16) but overlook that this applies as much to multiple regression as it does to bivariate regression. Multiple regression is blind to causation and even in very simple settings-if the goal is causal inference-"controlling for" variables can do more harm than good (see e.g., Achen, 2005; Cinelli et al., 2021). Indeed, naively reporting regression coefficients as "causal effects" is the well-known "Table 2" fallacy (Westreich and Greenland, 2013). In other words, bias in multiple regression can 
arise from both omitting variables (e.g., omitting a suspected confounding variable) and from including variables (e.g., (multi-)collinearity, collider bias, post-treatment bias, blocking a mediator).
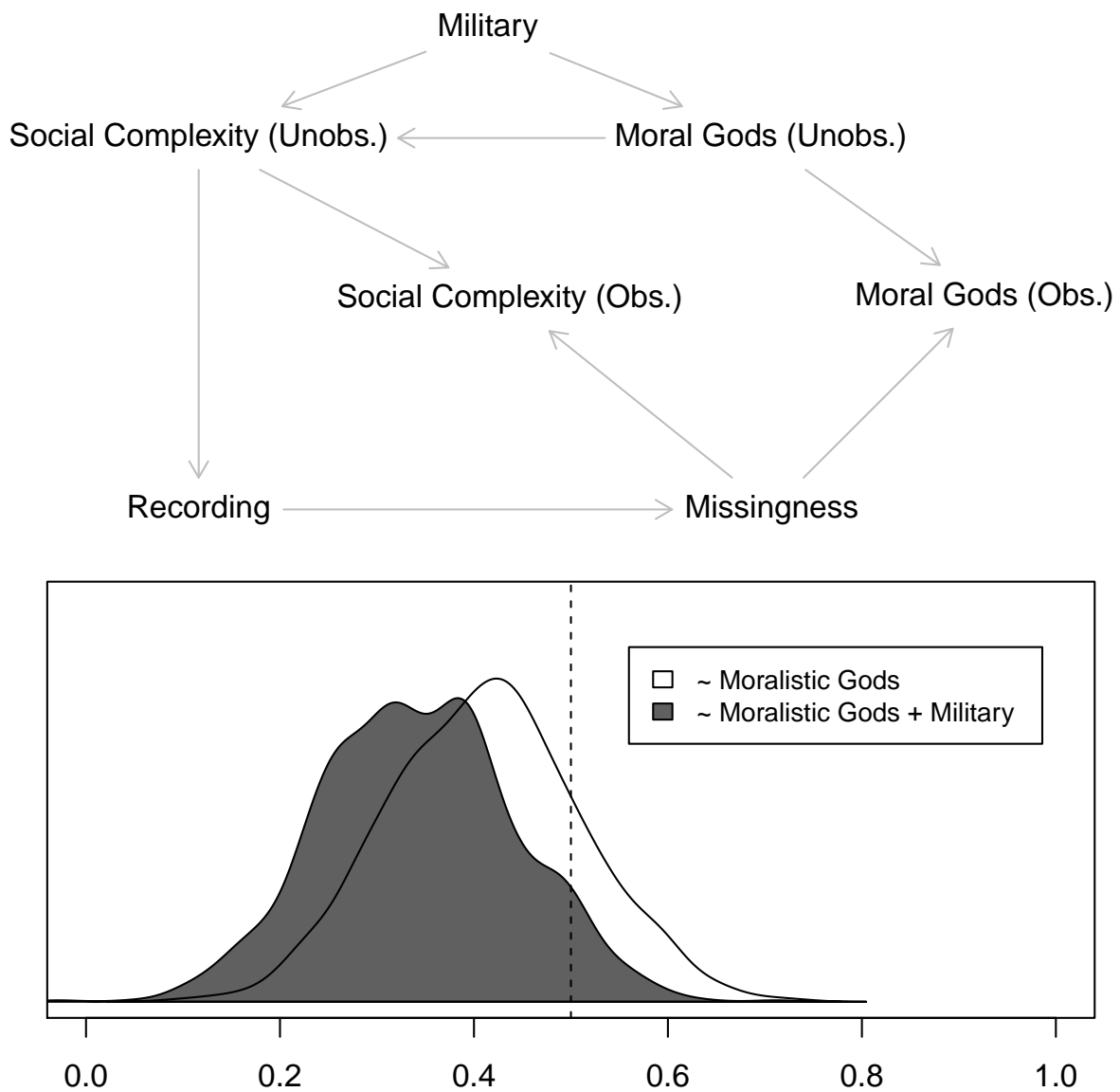

Figure 2: Upper: Causal model of data generation processes and target hypotheses. Lower: Distributions of 1,000 simulated estimates of observed moralistic gods' (MGO) effects on social complexity. All data $(n=100) \sim \operatorname{Normal}(0,1)$, all seed coefficients were set at 0.5 . For realism, we added some noise $\sim \operatorname{Normal}(0,1)$ to each variable.

Of course, by the time we get historical data, it has come to us through a set of filters, taphonomic processes, biases, historians, and so forth. These processes directly contribute to what observations we get to analyze. For inference-making, such metadata can therefore be just as important to consider as the data itself (Watts et al., in press). As mentioned earlier, some mechanism-in this case writing- 
is responsible for virtually all instances of "presence". Since we have neither written records nor a clear vision of what archaeological evidence of moralistic gods would look like for the past, the mechanism of evidence-provision causes the missingness or "inferred absence" values.

If we take the authors' focal prediction seriously, and model it with the process of historical recording and missingness in a causal graph, we see something along the lines of the upper panel of Fig. 2 (see also McElreath, 2020, 515). Here, the target causal estimand is the effect of true-but-unobserved moralistic gods on true-butunobserved social complexity. ${ }^{4}$ The available "test", however, is the effect of observed moralistic gods on observed social complexity. As unobserved social complexity contributes to the mechanism of recording history which in turn contributes to the availability of observations, we have a confounded causal model from above (military) and below (with the historical data generation process). According to the graph, there is no immediate way to obtain a reliable estimate of the target effect. Since we can't "control for" unobserved variables, recording, or missingness in this case, it is not a resolvable problem without blocking those paths with non-confounding exogenous variables.

To illustrate, we simulated the causal model and tested the empirically tractable hypothesis that observed moralistic gods predict observed social complexity (bottom panel of Fig. 2). Each coefficient-as represented by a causal arrow in the diagram-is set at 0.5 , the dotted line in the lower panel of Fig. 2. As we simulated each statistical model 1,000 times to produce Gaussian distributions around this value, the target estimate-the true-but-unobserved effect of moralistic gods on social complexity-should therefore be symmetrical around this line. The white distribution shows the estimated association between moralistic gods and social complexity without conditioning on any other variable, a clear underestimate of the true relationship. The gray distribution is the estimated association between moralistic gods and social complexity, but in a model that also holds military constant, showing an even smaller effect size of observed moralistic gods on observed social complexity, thus taking us even farther away from the true value. In other words, even though we know the target inference of the true-but-unobserved moralistic gods on truebut-unobserved social complexity is 0.5 , an analysis of observable data in this model does not give us an accurate inference. ${ }^{5}$

\footnotetext{
${ }^{4}$ Note that the following discussion holds if we create an unobserved counterpart for military, but we leave this out for the sake of clarity.

${ }^{5}$ In a regression output, the correct value for the effect of observed moralistic gods on observed social complexity while holding military constant should be $\approx 0.125$, since it would be the product
} 
In summary, the confluence of a) historical forces that gave rise to the kind of data available in the Seshat database, b) the coding decisions of that data, c) the subsequent analyses, and d) the lack of attention to a causal model prevents adequate tests of the target hypothesis. Again, the authors should be commended for their hard-work and adherence to open science practices. We appreciate the overarching goals of Seshat and acknowledge the massive effort required to compile and maintain a database. Using databases like Seshat to test hypotheses of this sort holds much promise. To live up to that promise, however, the serious flaws in their use - from data collection and coding to data transformation and analysis - have to be resolved before claiming to be able to reliably test target predictions.

\section{Acknowledgments}

We thank the Aarhus University Research Foundation for generous support. Purzycki acknowledges support from a Culture in Schooling grant funded by the Issachar Fund (\#TIF0206).

\section{References}

Achen, C. H. (2005). Let's put garbage-can regressions and garbage-can probits where they belong. Conflict Management and Peace Science, 22(4):327-339.

Beheim, B., Atkinson, Q. D., Bulbulia, J., Gervais, W., Gray, R. D., Henrich, J., Lang, M., Monroe, M. W., Muthukrishna, M., Norenzayan, A., Purzycki, B. G., Shariff, A., Slingerland, E., Spicer, R., and Willard, A. K. (2021). Treatment of missing data determined conclusions regarding moralizing gods. Nature, 595(7866):E29-E34.

Bendixen, T., Apicella, C., Atkinson, Q., Cohen, E., Henrich, J., McNamara, R. A., Norenzayan, A., Willard, A. K., Xygalatas, D., and Purzycki, B. G. (n.d.). Appealing to the minds of gods: A novel cultural evolutionary account of religious appeals and an empirical assessment using ethnographic data from eight diverse societies.

of the indirect effect between 1) observed and unobserved moralistic gods, 2) unobserved moralistic gods and unobserved social complexity, and 3) unobserved and observed social complexity (i.e., $0.5 *$ $0.5 * 0.5)$. However, because of the otherwise unblocked path through writing, the gray distribution is still biased. If we therefore hold missingness constant, we recover a range closer to 0.125 . 
Boehm, C. (2008). A biocultural evolutionary exploration of supernatural sanctioning. In Bulbulia, J., Sosis, R., Harris, Erica, Genet, R, and Wyman, K., editors, Evolution of Religion: Studies, Theories, and Critiques, pages 143-152. Collins Foundation Press, Santa Margarita, CA.

Cinelli, C., Forney, A., and Pearl, J. (2021). A crash course in good and bad controls. Available at SSRN $368943 \%$.

Evans-Pritchard, E. E. (1965). Theories of primitive religion. Oxford University Press, Oxford.

Gelman, A., Hwang, J., and Vehtari, A. (2014). Understanding predictive information criteria for bayesian models. Statistics and computing, 24(6):997-1016.

Hernán, M. A., Hernández-Díaz, S., Werler, M. M., and Mitchell, A. A. (2002). Causal knowledge as a prerequisite for confounding evaluation: an application to birth defects epidemiology. American journal of epidemiology, 155(2):176-184.

Lang, A. (1909). The making of religion. Longmans, London.

Liu, F. and Kong, Y. (2015). zoib: An R Package for Bayesian Inference for Beta Regression and Zero/One Inflated Beta Regression. The $R$ Journal, 7(2):34.

Malinowski, B. (1936). The Foundations of Faith and Morals. London: Oxford University Press UK.

McElreath, R. (2020). Statistical Rethinking: A Bayesian course with examples in $R$ and Stan. CRC Press, second edition.

Ospina, R. and Ferrari, S. L. P. (2012). A general class of zero-or-one inflated beta regression models. Computational Statistics \& Data Analysis, 56(6):1609-1623.

Pearl, J., Glymour, M., and Jewell, N. P. (2016). Causal inference in statistics: A primer. John Wiley \& Sons.

Purzycki, B. G. (2011). Tyvan cher eezi and the socioecological constraints of supernatural agents' minds. Religion, Brain $\&$ Behavior, 1(1):31-45.

Purzycki, B. G. (2013). The minds of gods: A comparative study of supernatural agency. Cognition, 129(1):163 - 179. 
Purzycki, B. G., Willard, A. K., Klocová, E. K., Apicella, C., Atkinson, Q., Bolyanatz, A., Cohen, E., Handley, C., Henrich, J., Lang, M., Lesorogol, C., Mathew, S., McNamara, R. A., Moya, C., Norenzayan, A., Placek, C., Soler, M., Weigel, J., Xygalatas, D., and Ross, C. T. (2022). The Moralization Bias of Gods' Minds: A Cross-Cultural Test. Religion, Brain and Behavior.

Singh, M., Kaptchuk, T. J., and Henrich, J. (2021). Small gods, rituals, and cooperation: The Mentawai water spirit Sikameinan. Evolution and Human Behavior, 42(1):61-72.

Swanson, G. E. (1960). The birth of the gods: The origin of primitive beliefs. University of Michigan Press.

Vehtari, A., Gelman, A., and Gabry, J. (2017). Practical Bayesian model evaluation using leave-one-out cross-validation and WAIC. Statistics and Computing, 27(5):1413-1432.

Watanabe, S. (2010). Asymptotic equivalence of bayes cross validation and widely applicable information criterion in singular learning theory. Journal of machine learning research, 11(12).

Watts, J., Greenhill, S. J., Atkinson, Q. D., Currie, T. E., Bulbulia, J., and Gray, R. D. (2015). Broad supernatural punishment but not moralizing high gods precede the evolution of political complexity in Austronesia. Proceedings of the Royal Society of London B: Biological Sciences, 282(1804):20142556.

Watts, J., Jackson, J. C., Arnison, C., Hamerslag, E., Shaver, J. H., and Purzycki, B. G. Building quantitative cross-cultural databases from ethnographic records: Promises, problems and principles. Cross-Cultural Research.

Westreich, D. and Greenland, S. (2013). The Table 2 Fallacy: Presenting and interpreting confounder and modifier coefficients. American journal of epidemiology, 177(4):292-298.

Whitehouse, H., François, P., Savage, P. E., Currie, T. E., Feeney, K. C., Cioni, E., Purcell, R., Ross, R. M., Larson, J., Baines, J., et al. (2021). Retraction note: Complex societies precede moralizing gods throughout world history. Nature, 595(7866):320-320. 